\title{
Emergency hospital admissions for psychiatric disorders in a German-wide hospital network during the COVID-19 outbreak
}

\author{
Jonathan Mathias Fasshauer ${ }^{1,2} \cdot$ Andreas Bollmann $^{3}$. Sven Hohenstein ${ }^{3} \cdot$ Gerhard Hindricks $^{3}$. \\ Andreas Meier-Hellmann ${ }^{4} \cdot$ Ralf Kuhlen $^{5} \cdot$ Andreas Broocks $^{6} \cdot$ Georg Schomerus ${ }^{2} \cdot$ Katarina Stengler $^{1}$
}

Received: 4 September 2020 / Accepted: 7 April 2021 / Published online: 17 April 2021

(c) Springer-Verlag GmbH Germany, part of Springer Nature 2021

\begin{abstract}
Purpose Psychiatric emergency hospital admissions for distinct psychiatric disorders and length of inpatient stay in the hospital during the Coronavirus disease 2019 (COVID-19) outbreak have not been thoroughly assessed.

Methods A retrospective study was performed analyzing claims data from a large German Hospital network during the COVID-19 outbreak (study period: March 13-May 21, 2020) as compared to periods directly before the outbreak (same year control: January 1-March 12, 2020) and one year earlier (previous year control: March 13-May 21, 2019).

Results A total of 13,151 emergency hospital admissions for psychiatric diagnoses were included in the analysis. For all psychiatric diagnoses combined, emergency admissions significantly decreased during the study period with mean (interquartile range) incidence rate ratios (IRRs) of $0.68(0.65,0.71)$ and $0.70(0.67,0.73)$ as compared to the same and previous year controls, respectively (both $p<0.00001$ ). IRR ranged from 0.56 for mood affective disorders (F30-F39) to 0.75 for mental disorders due to psychoactive substance use (F10-F19; all $p<0.00001)$. Mean (standard deviation) length of hospital stay for all psychiatric diagnoses was significantly shorter during the study period [9.8 (11.6) days] as compared to same [14.7 (18.7) days] and previous [16.4 (23.9) days] year controls (both $p<0.00001$ ).

Conclusion Both emergency hospital admissions and length of hospital stay significantly decreased for psychiatric disorders during the COVID-19 outbreak. It needs to be assessed in further studies whether healthcare systems will face increased demand for the provision of mental health care in the nearer future.
\end{abstract}

Keywords COVID-19 $\cdot$ Emergency admission $\cdot$ Length of stay $\cdot$ Psychiatric disorders

\section{Introduction}

Coronavirus disease 2019 (COVID-19) originated in Wuhan, China, in December 2019 and after worldwide spread WHO declared a pandemic on March 11, 2020 [1, 2]. On January

Katarina Stengler

katarina.stengler@helios-gesundheit.de

1 Department of Psychiatry, Psychotherapy and Psychosomatics, Helios Park Hospital Leipzig, Morawitzstraße 2, 04289 Leipzig, Germany

2 Department of Psychiatry, University of Leipzig, Leipzig, Germany

3 Heart Center Leipzig At University of Leipzig and Leipzig Heart Institute, Leipzig, Germany

4 Helios Hospitals, Berlin, Germany

5 Helios Health, Berlin, Germany

6 Department of Psychiatry and Psychotherapy, Carl-Friedric h-Flemming-Klinik, Schwerin, Germany 27, 2020, the first case of COVID-19 was officially confirmed in Germany [3]. It was the beginning of a pandemic outbreak with an increasing number of COVID-19 cases until May 2020 [4]. On March 13, the German government enforced broad restrictions including school and kindergarten closures, postponement of academic semesters, and prohibition of visits to nursing homes. Within 10 days, borders to neighboring countries were closed and strict social distancing measures were implemented within a partial lockdown. Only shops providing basic necessities including grocery stores and pharmacies remained open. The healthcare system prepared for increasing numbers of COVID-19 patients. Non-acute medical departments were repurposed 
for the management of COVID-19 and ventilator capacity was increased. Appointments for elective surgery were cancelled. Visiting of patients in hospitals was prohibited with exceptions including dying patients [4]. Extensive psychological stress for the medical staff has also been observed [5].

The partial lockdown measures initiated on March 13 and formally implemented German-wide on March 22 [6] could have affected emergency care for psychiatric patients in two opposing ways: on one hand, mental distress caused by isolation, lack of outpatient treatment, and non-availability of supporting treatment groups might adversely affect mental health causing greater demand for emergency health care provision [7]. On the other hand, fear of COVID-19 infection in hospitals might prevent patients from using health care services as has been shown by our group for heart failure and cardiac arrhythmias [8]. Furthermore, shifting of health care resources towards the therapy of COVID-19 might decrease treatment capacity for non-intensive care in general and psychiatric disorders in particular. Several studies indicate that emergency presentations for mental health conditions decreased during the COVID-19 pandemic worldwide [9-14]. However, the impact of COVID-19 on emergency hospital admissions and length of hospital stay for defined psychiatric disorders remains unclear. Furthermore, differences between the early and late phase of the partial lockdown have not been assessed so far. To address these open points, data from 67 hospitals of a large and regionally diverse German health care provider ("Helios hospitals") were evaluated concerning number of admissions for psychiatric disorders and length of hospital stay.

\section{Methods}

\section{Study cohort with inclusion and exclusion criteria}

A retrospective analysis of claims data from 67 Helios hospitals was performed. Inclusion criteria were emergency admissions and International Statistical Classification of Diseases and Related Health Problems (ICD) code of main diagnoses F00-F69. These ICD codes comprised organic, including symptomatic, mental disorders (F00-F09), mental and behavioral disorders due to psychoactive substance use (F10-F19), schizophrenia, schizotypal, and delusional disorders (F20-F29), mood [affective] disorders (F30-F39), neurotic, stress-related, and somatoform disorders (F40F48), behavioral syndromes associated with physiological disturbances and physical factors (F50-F59), and disorders of adult personality and behavior (F60-F69). Cases with lab-confirmed COVID-19 infection (ICD code U07.1) were excluded. Administrative data were extracted from QlikView (QlikTech, Radnor, Pennsylvania, USA). Data were stored in a pseudonomized form. This study was approved by the Ethics Committee at the Medical Faculty, Leipzig University (\#490/20-ek). Due to the retrospective study of anonymized data, informed consent was not obtained.

\section{Study periods}

Three periods were defined to compare emergency hospital admissions and length of stay for defined psychiatric diagnoses. The study period (March 13-May 21, 2020) was compared to two control periods, i.e. directly before the outbreak (January 1-March 12, 2020; same year control) and one year earlier (March 13-May 21, 2019; previous year control). For further analyses, the study period was subdivided into an early (March 13-April 10, 2020) and late (April 11-May $21,2020)$ phase of the COVID-19 outbreak with the nadir of daily F00-F69 admissions in 2020 defined as the border.

\section{Statistical analyses}

Incidence rate (IR) values for admissions were calculated by dividing the number of cumulative admissions for each of the psychiatric diagnoses groups by the number of days for each time period. IR ratio (IRR) values comparing the study period to each of the control periods were calculated using Poisson generalized linear mixed models (GLMM) with log link function for count data and specifying hospitals as random factor to model the number of cause-specific emergency hospitalizations per day $[15,16]$. Effects were estimated with the lme4 package (version 1.1-21) [17] in the $\mathrm{R}$ environment for statistical computing (version 3.6.1, 64-bit build) [18]. In all models, varying intercepts for the random factor were specified. The IRR values for the different factor levels are based on different models comparing the periods. They were calculated by exponentiation of the negative of the regression coefficients together with $95 \%$ confidence intervals for the comparisons of the different periods and $p$ values for the interactions are reported. For all tests, a two-tailed 5\% error criterion for significance was applied. Analysis of the outcome variable length of stay was performed via Poisson GLMM. Means, standard deviations (SDs), ratios, 95\% confidence intervals, and $p$ values are reported.

\section{Results}

\section{Admissions per day: study period vs. control periods}

A total of 13,151 emergency hospital admissions for the psychiatric diagnoses studied were included in the analysis consisting of 3327 (IR of 47.5) admission in the study period, as well as 5049 (IR of 70.1) and 4775 (IR of 68.2) 
admissions in the same and previous year control periods, respectively (Table 1). Mean age and sex distribution were similar in the study period as compared to both control periods (Table 1). For all psychiatric diagnoses, emergency admissions significantly declined during the study period with IRRs of $0.68(0.65,0.71)$ and $0.70(0.67$, 0.73 ) as compared to the same and previous year controls, respectively (both $p<0.00001$; Table 1 ). Decreases in emergency admissions were statistically significant for all psychiatric diagnoses studied and IRRs ranged from 0.56 (F30-F39 study period as compared to same year control) to 0.75 (F10-F19 study period as compared to previous year control) (all $p<0.00001$; Table 1 ).
Table 1 Emergency hospital daily admissions (IRR) for psychiatric diagnoses in the German-wide Helios hospital network between the COVID19 outbreak and two control periods

\begin{tabular}{|c|c|c|c|}
\hline Psychiatric diagnoses ICD-10 & Study period & Same year control & Previous year control \\
\hline \multicolumn{4}{|l|}{ Combined (F00-F69) } \\
\hline Age (years) & $46.1(21.2)$ & $45.4(21.6)$ & $45.8(21.7)$ \\
\hline Female & $1364(41)$ & $2233(44)$ & $2059(43)$ \\
\hline No. of cumulative admissions & 3327 & 5049 & 4775 \\
\hline No. of daily admissions (IR) & 47.5 & 70.1 & 68.2 \\
\hline IRR & & $0.68(0.65,0.71)$ & $0.70(0.67,0.73)$ \\
\hline$p$ value & & $<0.00001$ & $<0.00001$ \\
\hline \multicolumn{4}{|l|}{ F00-F09 } \\
\hline Age (years) & $79.3(11.6)$ & $77.5(14.6)$ & $78.2(11.8)$ \\
\hline Female & $194(53)$ & $279(51)$ & $286(54)$ \\
\hline No. of cumulative admissions & 368 & 551 & 532 \\
\hline No. of daily admissions (IR) & 5.3 & 7.7 & 7.6 \\
\hline IRR & & $0.69(0.60,0.78)$ & $0.69(0.61,0.79)$ \\
\hline$p$ value & & $<0.00001$ & $<0.00001$ \\
\hline \multicolumn{4}{|l|}{ F10-F19 } \\
\hline Age (years) & $42.5(16.5)$ & $40.9(16.8)$ & $41.5(17.1)$ \\
\hline Female & $399(24)$ & $651(28)$ & $594(27)$ \\
\hline No. of cumulative admissions & 1640 & 2298 & 2192 \\
\hline No. of daily admissions (IR) & 23.4 & 31.9 & 31.3 \\
\hline IRR & & $0.73(0.69,0.78)$ & $0.75(0.70,0.80)$ \\
\hline$p$ value & & $<0.00001$ & $<0.00001$ \\
\hline \multicolumn{4}{|l|}{ F30-F39 } \\
\hline Age (years) & $43.1(21.6)$ & $44.5(22.0)$ & $46.7(22.0)$ \\
\hline Female & $272(64)$ & $478(61)$ & $391(58)$ \\
\hline No. of cumulative admissions & 425 & 786 & 675 \\
\hline No. of daily admissions (IR) & 6.1 & 10.9 & 9.6 \\
\hline IRR & & $0.56(0.49,0.63)$ & $0.63(0.56,0.71)$ \\
\hline$p$ value & & $<0.00001$ & $<0.00001$ \\
\hline \multicolumn{4}{|l|}{ F40-F48 } \\
\hline Age (years) & $40.6(21.8)$ & $40.2(22.3)$ & $38.2(22.0)$ \\
\hline Female & $261(59)$ & $447(63)$ & $466(65)$ \\
\hline No. of cumulative admissions & 444 & 712 & 721 \\
\hline No. of daily admissions (IR) & 6.3 & 9.9 & 10.3 \\
\hline IRR & & $0.64(0.57,0.72)$ & $0.62(0.55,0.69)$ \\
\hline$p$ value & & $<0.00001$ & $<0.00001$ \\
\hline
\end{tabular}

Age is presented as mean (SD), IRR as mean (95\% confidence interval), and female sex as number (percentage)

ICD codes represent: organic, including symptomatic, mental disorders (F00-F09), mental and behavioral disorders due to psychoactive substance use (F10-F19), schizophrenia, schizotypal, and delusional disorders (F20-F29), mood [affective] disorders (F30-F39), neurotic, stress-related, and somatoform disorders (F40-F48), behavioral syndromes associated with physiological disturbances and physical factors (F50F59), and disorders of adult personality and behavior (F60-F69)

$I C D-10$ International classification of diseases, $I R$ incidence rate, $I R R$ incidence rate ratio, No number 


\section{Admissions per day: late vs. early study period}

When further subdividing the study period, cumulative number of admissions was 1361 (IR of 46.9) for the early phase and 1966 (IR of 48.0) for the late phase (Supplemental Table 1). Mean age and sex distribution were similar between both study periods (Supplemental Table 1). For all psychiatric diagnoses, daily admissions were not significantly different between the early and late study period with an IRR of $0.98(0.91,1.05 ; p=0.540$; Supplemental Table 1). Results were heterogeneous for specific psychiatric diagnosis groups. Thus, daily admissions were significantly lower in the early as compared to the late study period for F40-F48 ( $p=0.011$; Supplemental Table 1). In contrast, there was a trend towards higher number of daily admissions in the early as compared to the late period for F30-F39 $(p=0.050$; Supplemental Table 1).

\section{Length of hospital stay: study period vs. controls}

For all psychiatric diagnoses, length of stay was significantly shorter during the study period [9.8 (11.6)] as compared to the same [14.7 (18.7)] and previous [16.4 (23.9)] year control, respectively (both $p<0.00001$; Table 2). Ratios between length of stay of the study period and the control periods were statistically significant for all psychiatric diagnoses studied and ranged from 1.19 [(1.14, 1.23); F00-F09 study period as compared to same year control] to 1.74 $[(1.69,1.79)$; F30-F39 study period as compared to previous year control] (all $p<0.0001$; Table 2).

\section{Length of hospital stay: late vs. early study period}

For all psychiatric diagnoses, length of stay was significantly higher during the early [12.4 (14.7)] as compared to the late [8.0 (8.4)] study period $(p<0.00001$; Supplemental Table 2). Ratios between early and late study period were statistically significant for all psychiatric diagnoses studied and ranged from 0.63 [(0.60, 0.66); F30-F39] to 0.82 [(0.78, 0.85); F10-F19) (all $p<0.00001$; Supplemental Table 2).

\section{Discussion}

\section{Hospital admissions}

In the current study, it is shown that the number of emergency hospital admissions significantly decreased and hospital length of stay was significantly shorter during the partial COVID-19 lockdown in Germany for all psychiatric disorders as compared to two control periods. Emergency admissions decreased for all psychiatric diagnosis groups with IRRs ranging from 0.56 for mood (affective) disorders
Table 2 Length of stay of emergency hospital admissions for psychiatric diagnoses in the German-wide Helios hospital network between the COVID-19 outbreak and two control periods

\begin{tabular}{|c|c|c|c|}
\hline $\begin{array}{l}\text { Psychiatric } \\
\text { diagnoses } \\
\text { ICD-10 }\end{array}$ & Study period & $\begin{array}{l}\text { Same year } \\
\text { control }\end{array}$ & $\begin{array}{l}\text { Previous year } \\
\text { control }\end{array}$ \\
\hline \multicolumn{4}{|c|}{ Combined (F00-F69) } \\
\hline Age (years) & $46.1(21.2)$ & $45.4(21.6)$ & $45.8(21.7)$ \\
\hline Female & $1,364(41)$ & $2,233(44)$ & $2,059(43)$ \\
\hline Length of stay & $9.8(11.6)$ & $14.7(18.7)$ & $16.4(23.9)$ \\
\hline Ratio & & $1.51(1.49,1.53)$ & $1.68(1.66,1.71)$ \\
\hline$p$ value & & $<0.00001$ & $<0.00001$ \\
\hline \multicolumn{4}{|l|}{ F00-F09 } \\
\hline Age (years) & $79.3(11.6)$ & $77.5(14.6)$ & $78.2(11.8)$ \\
\hline Female & $194(53)$ & $279(51)$ & $286(54)$ \\
\hline Length of stay & $13.0(11.2)$ & $14.9(15.5)$ & $15.9(19.3)$ \\
\hline Ratio & & $1.19(1.14,1.23)$ & $1.25(1.20,1.30)$ \\
\hline$p$ value & & $<0.00001$ & $<0.00001$ \\
\hline \multicolumn{4}{|l|}{ F10-F19 } \\
\hline Age (years) & $42.5(16.5)$ & $40.9(16.8)$ & $41.5(17.1)$ \\
\hline Female & $399(24)$ & $651(28)$ & $594(27)$ \\
\hline Length of stay & $6.0(7.2)$ & $7.7(9.9)$ & $7.6(10.6)$ \\
\hline Ratio & & $1.31(1.28,1.35)$ & $1.29(1.25,1.32)$ \\
\hline$p$ value & & $<0.00001$ & $<0.00001$ \\
\hline \multicolumn{4}{|l|}{ F30-F39 } \\
\hline Age (years) & $43.1(21.6)$ & $44.5(22.0)$ & $46.7(22.0)$ \\
\hline Female & $272(64)$ & $478(61)$ & $391(58)$ \\
\hline Length of stay & $17.3(15.2)$ & $25.8(23.4)$ & $30.5(28.6)$ \\
\hline Ratio & & $1.49(1.45,1.53)$ & $1.74(1.69,1.79)$ \\
\hline$p$ value & & $<0.00001$ & $<0.00001$ \\
\hline \multicolumn{4}{|l|}{ F40-F48 } \\
\hline Age (years) & $40.6(21.8)$ & $40.2(22.3)$ & $38.2(22.0)$ \\
\hline Female & $261(59)$ & $447(63)$ & $466(65)$ \\
\hline Length of stay & $6.7(9.0)$ & $10.2(15.5)$ & $10.0(19.0)$ \\
\hline Ratio & & $1.57(1.50,1.64)$ & $1.54(1.47,1.61)$ \\
\hline$p$ value & & $<0.00001$ & $<0.00001$ \\
\hline
\end{tabular}

Age is presented as mean (SD), length of stay is presented as mean (SD), ratio as mean (95\% confidence interval), and female sex as number (percentage)

ICD codes represent: organic, including symptomatic, mental disorders (F00-F09), mental and behavioral disorders due to psychoactive substance use (F10-F19), schizophrenia, schizotypal, and delusional disorders (F20-F29), mood [affective] disorders (F30-F39), neurotic, stress-related, and somatoform disorders (F40-F48), behavioral syndromes associated with physiological disturbances and physical factors (F50-F59), and disorders of adult personality and behavior (F60F69)

ICD-10 International Classification of Diseases, $I R$ incidence rate, $I R R$ incidence rate ratio, No number

to 0.75 for mental and behavioral disorders due to psychoactive substance use. Overall number of emergency psychiatric admissions was not significantly different between the early and late study period. In agreement with our findings, 
emergency admissions for affective disorders were significantly reduced by $55 \%$ in an independent study from Italy [9]. Admission rates were numerically but not significantly reduced for other psychiatric diagnoses, i.e. schizophrenia spectrum disorders, personality disorders, and substance use disorders in the study by Clerici and co-workers [9]. The lower number of psychiatric admissions during the COVID-19 lockdown analyzed $(n=182)$ [9] as compared to the current study period $(n=3327)$ might well explain the different findings. Total emergency hospital admissions have also decreased during the local COVID-19 outbreak in Connecticut, USA [10]. Furthermore, the rate of hospitalization of psychiatric patients was slightly higher as compared to the control period [10]. Similar findings were obtained in an independent study from France also showing a decreased number of hospital admissions combined with a slight increase in hospitalization rate [11]. Other studies have evaluated emergency presentations but not emergency admissions for psychiatric disorders during the COVID-19 lockdown. Absolute number of psychiatric presentations was not altered in a study from Germany [12]. However, the share of psychiatric emergency presentations increased due to a decrease in the total number of emergency cases [12]. In contrast, psychiatric emergency service cases during COVID-19 decreased by $27 \%$ in another study from Germany [13]. Similarly, mean number of emergency presentations for psychiatric disorders were more than $50 \%$ reduced in a study from France [14]. On a broader perspective, psychiatric outpatient cases in Germany decreased up to 39.8\% at the end of March with some recovery effect starting at the end of May 2020 as compared to the previous year [19]. Taking the current results and published evidence into consideration, emergency presentations, hospital admissions, and outpatient care all decreased during the COVID-19 pandemic over the whole spectrum of psychiatric diagnoses.

Uncertainty of the pandemic, as well as associated lockdowns, physical distancing, and economic downturn could increase the risk of mental health problems [20, 21]. In agreement with this notion, topics for COVID-19-related medical consultations included not only fear of COVID19 infection but also stress due to visiting bans and social distancing measures, as well as socioeconomic problems, in a German study [12]. Studies elucidating the impact of COVID-19 on suicide rates have yielded mixed results. In a study from Japan, monthly suicide rates declined by $14 \%$ during the first 5 months of the pandemic but increased by $16 \%$ thereafter [22]. In contrast, suicide attempts were more frequent ( $22 \%$ vs. $6 \%$ ) as compared to controls without COVID-19-related consultations in a study from Germany [12]. In a case from the Department of Psychiatry of the University of Leipzig, the impact of the pandemic intensified the delusional disorder of a woman and led to an attempted suicide [23]. No clear changes in suicide rates were seen in England between January and September 2020 with fluctuation of numbers rather due to delays of the coroner's service [24].

The course of psychiatric disorders in patients with indication for emergency psychiatric admission but not presenting in the emergency room needs to be assessed in further studies. On the one hand, the majority of these patients remained without professional support possibly worsening their mental health conditions. It is important to note in this context that non-hospital care options in Germany were also restricted during the first lockdown with psychiatric outpatient cases decreasing by $39.8 \%$ at the end of March 2020 and recovery effects not seen before end of May 2020 as compared to the previous year [19]. On the other hand, the pandemic could have curtailed non-essential bed use in treating people with psychiatric disorders. It needs to be emphasized in this context that psychiatric care beds in hospitals per 100,000 inhabitants in 2017 were more than tenfold higher in Germany as compared to Italy, i.e. 128 versus 9 [25]. Clearly, more work is needed to elucidate medium- to long-term effects of the COVID-19 pandemic on mental health care worldwide.

\section{Length of stay}

In the current study, length of hospital stay for emergency psychiatric admissions was also determined. Clerici and co-workers demonstrate convincingly that median time of hospitalization significantly increased from 10 days in 2019 to 14 days during COVID-19 in 2020 [9]. In contrast, length of hospital stay was significantly reduced for all psychiatric diagnosis groups examined in the current study. The discrepant results might be well explained by the different patient populations and time periods studied.

It is interesting to note in this context that hospital length of stay was significantly higher in the early as compared to the late study period for all psychiatric diagnoses studied in the current report. Since hospitals emerged as a source of many COVID-19 infections, a growing notion of the dangers of nosocomial infection might have contributed to the shortening of inpatient stays over time. Furthermore, patients remaining in hospital during the early phase of the lockdown might have been more severely affected by mental health problems as compared to the later phase. In addition, patients in the early phase of the pandemic might have felt secure in the hospital due to high hygienic standards and frequent smear tests. However, no data are available in the currently studied cohort to assess these hypotheses. Parallel reduction of new admissions combined with less pressure to discharge inpatients as suggested by Clerici and co-workers [9] might also contribute. 


\section{Strengths and limitations}

Strengths of the study include the large sample size from a regionally diverse German hospital network. Limitations include the retrospective nature of the analysis. Furthermore,

data from an administrative, multi-center dataset were studied which were not stored for research purposes but for remuneration reasons. This could potentially affect the encoded information [26] and quality of the results depends to a large extent on the correct encoding of ICD-codes [27]. Moreover, patient outcome data are not available in the pseudonymized data set used for the current study.

\section{Conclusions}

Taken together, the current study demonstrates that both emergency hospital admissions and length of hospital stay significantly decreased for a broad range of psychiatric disorders during the COVID-19 outbreak in Germany. Increased length of hospital stay during the early as compared to the late phase of the outbreak might be due to a growing notion of the dangers of nosocomial infection over time and more severe disease in affected patients. It needs to be assessed in further studies whether health-care systems will face increased demand for provision of mental health care in the nearer future.

Supplementary Information The online version contains supplementary material available at https://doi.org/10.1007/s00127-021-02091-z.

\begin{abstract}
Authors' contributions All authors contributed to the study conception and design. Material preparation, data collection and analysis were performed by JMF, AB, SH, GS, and KS. The first draft of the manuscript was written by JMF, GS, and KS and all authors commented on previous versions of the manuscript. All authors read and approved the final manuscript.
\end{abstract}

Funding This study received no specific funding.

Availability of data and material Restrictions apply due to data protection laws.

Code availability Data were analyzed using R software version 3.6.1. Statistical code generating the results of this manuscript can be requested from the authors.

\section{Declarations}

Conflict of interest The authors declare no conflict of interest.

Ethics approval This study was approved by the Ethics Committee at the Medical Faculty, Leipzig University (\#490/20-ek).

Consent to participate Due to the retrospective study of anonymized data, informed consent was not obtained.

\section{References}

1. Zhu N, Zhang D, Wang W et al (2020) A novel Coronavirus from patients with pneumonia in China, 2019. N Engl J Med 382:727-733. https://doi.org/10.1056/NEJMoa2001017

2. WHO Director General. Opening remarks at the media briefing on COVID-19. Im Internet: https://www.who.int/dg/speeches/ detail/who-director-general-s-opening-remarks-at-the-mediabriefing-on-covid-19---11-march-2020. Accessed 01 Aug 2020

3. Böhmer MM, Buchholz U, Corman VM et al (2020) Investigation of a COVID-19 outbreak in Germany resulting from a single travel-associated primary case: a case series. Lancet Infect Dis 20:920-928. https://doi.org/10.1016/S1473-3099(20) 30314-5

4. Carroll WD, Strenger V, Eber E et al (2020) European and United Kingdom COVID-19 pandemic experience: the same but different. Paediatr Respir Rev 35:50-56. https://doi.org/10. 1016/j.prrv.2020.06.012

5. Bohlken J, Schömig F, Lemke MR et al (2020) COVID-19 pandemic: stress experience of healthcare workers-a short current review. Psychiat Prax 47:190-197. https://doi.org/10. 1055/a-1159-5551

6. Meeting between the Chancellor and the heads of government of the federal states (German). Internet: https://www.bundesregi erung.de/breg-de/themen/coronavirus/besprechung-der-bunde skanzlerin-mit-den-regierungschefinnen-und-regierungschefsder-laender-1733248. Accessed 09 Aug 2020

7. Richter D, Zürcher S (2020) Mental health care services during the COVID-19 pandemic. Psychiat Prax 47:173-175. https:// doi.org/10.1055/a-1157-8508

8. Bollmann A, Hohenstein S, Meier-Hellmann A et al (2020) Emergency hospital admissions and interventional treatments for heart failure and cardiac arrhythmias in Germany during the COVID-19 outbreak: insights from the German-wide Helios hospital network. Eur Heart J Qual Care Clin Outcomes 6:221222. https://doi.org/10.1093/ehjqcco/qcaa049

9. Clerici M, Durbano F, Spinogatti F et al (2020) Psychiatric hospitalization rates in Italy before and during COVID-19: did they change? An analysis of register data. Ir j psychol Med. https://doi.org/10.1017/ipm.2020.29

10. Goldenberg MN, Parwani V (2020) Psychiatric emergency department volume during COVID-19 pandemic. Am J Emerg Med. https://doi.org/10.1016/j.ajem.2020.05.088

11. Pignon B, Gourevitch R, Tebeka S et al (2020) Dramatic reduction of psychiatric emergency consultations during lockdown linked to COVID-19 in Paris and suburbs. Psychiatry Clin Neurosci. https://doi.org/10.1111/pen.13104

12. Aly L, Sondergeld R, Hölzle P et al (2020) The COVID-19 pandemic has not changed the number but the type of psychiatric emergencies: a comparison of care data between 2019 and 2020. Nervenarzt 24:1-3. https://doi.org/10.1007/s00115-020-00973-2

13. Hoyer C, Ebert A, Szabo K et al (2020) Decreased utilization of mental health emergency service during the COVID-19 pandemic. Eur Arch Psychiatry Clin Neurosci 9:1-3. https://doi. org/10.1007/s00406-020-01151-w

14. Pham-Scottez A, Silva J, Barruel D et al (2020) Patient flow in the largest French psychiatric emergency centre in the context of the COVID-19 pandemic. Psychiatry Res 291:113205. https:// doi.org/10.1016/j.psychres.2020.113205

15. Baayen RH, Davidson DJ, Bates DM (2008) Mixed-effects modeling with crossed random effects for subjects and items. J Mem Lang 59:390-412. https://doi.org/10.1016/j.jml.2007.12.005

16. Kliegl R, Masson MEJ, Richter EM (2010) A linear mixed model analysis of masked repetition priming. Vis Cogn 18:655681. https://doi.org/10.1080/13506280902986058 
17. Bates D, Mächler M, Bolker B et al (2015) Fitting linear mixedeffects models using lme4. J Stat Softw 67:1-48. https://doi.org/ 10.18637/jss.v067.i01

18. R Core Team (2020) $\mathrm{R}$ a language and environment for statistical computing. Internet: https://www.R-project.org/https://www.Rproject.org/. Accessed $25 \mathrm{Jul} 2020$

19. Mangiapane DS, Zhu L, Czihal T. Change in utilization of services by primary care physicians during the COVID crisis (German). Im Internet: https://www.zi.de/fileadmin/images/conte nt/Publikationen/Trendreport_3_Leistungsinanspruchnahme_ COVID_final.pdf. Accessed 13 Feb 2021

20. Moreno C, Wykes T, Galderisi S et al (2020) How mental health care should change as a consequence of the COVID-19 pandemic. Lancet Psychiatry 7:813-824. https://doi.org/10.1016/S22150366(20)30307-2

21. Bojdani E, Rajagopalan A, Chen A et al (2020) COVID-19 pandemic: impact on psychiatric care in the United States. Psychiatry Res 289:113069. https://doi.org/10.1016/j.psychres.2020.113069

22. Tanaka T, Okamoto S (2021) Increase in suicide following an initial decline during the COVID-19 pandemic in Japan. Nat Hum Behav. https://doi.org/10.1038/s41562-020-01042-Z
23. Weise J, Schomerus G, Speerforck S (2020) Pandemic and an attempted suicide of a patient with delusional disorder. Psychiat Prax 47:218-220. https://doi.org/10.1055/a-1158-1745

24. Nasir R, Manders B. Quarterly suicide death registrations in England-Office for National Statistics. Internet: https://www.ons. gov.uk/peoplepopulationandcommunity/birthsdeathsandmarri ages/deaths/bulletins/quarterlysuicidedeathregistrationsinengl and/2001to2019registrationsandquarter1jantomartoquarter3jul ytosept2020provisionaldata. Accessed 13 Feb 2021

25. Eurostat. Hospital beds by type of care. Im Internet: https:// appsso.eurostat.ec.europa.eu/nui/show.do?dataset=hlth_rs_bds\& lang=en. Accessed 13 Feb 2021

26. Administrative SR, Data S (2016) Potential and pitfalls. J Invest Dermatol 136:2122-2124. https://doi.org/10.1016/j.jid.2016.07. 022

27. Rangachari P (2007) Coding for quality measurement: the relationship between hospital structural characteristics and coding accuracy from the perspective of quality measurement. Perspect Health Inf Manag 4:3 